\title{
LA TRANSFERENCIA DE POLÍTICAS GLOBALES DE GÉNERO Y SU PUESTA EN AGENDA
}

Paula Ruiz*

Resumen

Desde la década de los años ochenta, el género se incorpora como categoría analítica e interpretativa que ha venido evolucionado tanto en su desarrollo teórico y metodológico como político y social. En el ámbito académico, por ejemplo, su análisis se ha abordado como una construcción cultural y social, lo cual ha permitido ampliar su espacio de discusión y de estudio como método de investigación dentro de las ciencias sociales. El feminismo, como movimiento social, ha impactado en la construcción de políticas en pro del empoderamiento de la mujer, impulsando a los Estados a suscribir acuerdos que paulatinamente, en la mayoría de sociedades, han mejorado su situación en los distintos ámbitos. La movilización internacional sobre temas blandos de la agenda internacional ha llevado a la trans- ferencia de politicas de un sistema a otro y a su puesta en agenda a nivel local. En este artículo se estudiarán los aportes que el feminismo ha desarrollado en materia de investigación dentro de las relaciones internacionales, así como la influencia que el género ha tenido para la formulación de políticas globales y la implementación de estas a nivel local.

Palabras clave: transferencia de políticas, género, feminismo, relaciones internacionales.

\section{THE TRANSFER OF GLOBAL GENDER POLITICS AND ITS SETTING ONTHE AGENDA}

\begin{abstract}
Since the eighties, the gender issue has been evolved into the theoretical and methodological development as same as the
\end{abstract}

* Estudiante de Doctorado en Estudios Políticos. Coordinadora Académica, Especialización en Cooperación Internacional y Gestión de Proyectos para el Desarrollo, Universidad Externado de Colombia, Bogotá (Colombia). paula.ruiz@uexternado.edu.co

Recibido: 6 de marzo de 2015 / Modificado: 13 de abril de 2015 / Aceptado: 22 de abril de 2015.

Para citar este artículo

Ruiz, P. (2015). La transferencia de políticas globales de género y su puesta en agenda. OPERA, 16, pp. 55-75. DOI: $10.18601 / 16578651 . n 16.05$ 
political and social debate. Inside academic research, political science and international relations have addressed this issue as a cultural and social construction, expanding its space for discussion and study as a research method in the social sciences. Feminism, as a social movement, has impacted the construction of international policies for promoting women's empowerment. States sign agreements that gradually, in most societies, have improved the women status. International mobilization on soft issues on the international agenda has led to policy transfer from one system to another and putting it on the agenda at the local level. Therefore, in this article the contributions on Feminism and International Relations has developed, as the influence that gender has had on global policy making and implementation at the local level will be studied.

Key words: Policy transfer, gender, feminism, international relations.

\section{INTRODUCCIÓN}

"Las mujeres tienen menos oportunidades que los hombres de vivir libres de temores y de disfrutar de tipos más gratificantes de amor" (Nussbaum, 2002, p. 28).
Una preocupación central sobre el género, entendido como categoría analítica, es que a pesar de todos los grandes avances que desde distintos ámbitos (global, nacional, local) se realizan, la paridad entre hombres y mujeres no se ha alcanzado en algunos países en desarrollo en campos como la educación, la salud o el empleo ${ }^{1}$. Sin embargo, la construcción de políticas globales, la implementación de programas en el ámbito local y los grandes aportes que desde el punto de vista de la investigación social y de la academia se realizan, han llevado a la movilización de distintos actores que claman por acciones más decididas y efectivas por parte de sus gobernantes para alcanzar la igualdad de género en todos los ámbitos.

El tema sobre la participación política de las mujeres, por ejemplo, ha tenido una fuerte influencia en la comunidad internacional en el seno de la Organización de las Naciones Unidas (ONU), desde donde se ha venido promoviendo un trato político igualitario, así como el derecho de las mujeres a intervenir en asuntos políticos.

Las mujeres y sus intereses políticos han sido marginalizados como resultado de unas históricas estructuras de Estado y de sus instituciones políticas, privilegiando a los hombres, sus voces y sus decisiones. Durante el último siglo, las mujeres han ido gradualmente incrementando

1 Según el Informe 2014 del Programa de las Naciones Unidas para el Desarrollo (PNUD), en el cual se hace un balance sobre el cumplimiento de los Objetivos de Desarrollo del Milenio a nivel mundial, en regiones como África subsahariana, Oceanía y Asia Occidental, las niñas aún se enfrentan a muchos obstáculos para poder acceder a la educación primaria y secundaria. En regiones como África septentrional, solo una de cada cinco mujeres recibe remuneración por su trabajo en el sector agrícola (PNUD, 2014, p. 20). 
su participación en política como votantes, tomadoras de decisión y como miembros de Organizaciones No Gubernamentales. La agenda global ha sido testigo de las discriminaciones y ha buscado mitigar las desigualdades en términos de género ${ }^{2}$ (Staudt, 2008, p. 162).

En el marco del estudio de las relaciones internacionales, las políticas de género han sido abordadas como parte de los temas blandos de la agenda internacional ${ }^{3}$, al igual que aquellos relacionados con la protección de minorías, desarrollo social o cultural. Estos temas blandos no son prioritarios para los Estados, como sí lo son aquellos susceptibles de representar algún tipo de amenaza para la paz y la seguridad internacional, por ejemplo, el terrorismo o el uso de armas químicas y nucleares que se consideran temas duros.

No obstante, igualar la construcción y legitimación de los temas de género respecto a otros temas blandos es objeto de discusión y de análisis desde el feminismo. Para Marta Ochman, la discriminación o inequidad que enfrentan las mujeres no es la misma que deben enfrentar las minorías nacionales, los pobres o los desempleados (2006, p. 386).

La construcción académica alrededor del concepto de género ha sido uno de los aportes más significativos del feminismo. "Tanto la noción de lo femenino como de lo masculino son el resultado de construcciones sociales y no de hechos naturales o biológicos" (Cobo, 1995 , p. 55) y, por tanto, se busca poner de manifiesto cuál es el rol que la mujer ha venido ejerciendo a lo largo de la historia y en distintos campos. "Esos nuevos modos de análisis exigen el uso de la investigación, el análisis y el razonamiento para emprender una reflexión en torno al lugar y el peso de la diferencia (raza, género, sexualidad, clase) en nuestra teorización" (Wallerstein, 2006, p. 61).

Lo anterior, es el resultado de "voces disidentes que cuestionaron la capacidad de las ciencias sociales para explicar la realidad de ellas. Parecían decir a los investigadores: es posible que tu análisis sea apropiado para tu grupo, pero simplemente no encaja en $\mathrm{mi}$ caso" (Wallerstein, 2006, p. 56). Esto ilustra lo que al interior de los movimientos feministas $y$, posteriormente, entre algunos académicos pasaba durante la década de los ochenta.

A partir de la década de los setenta, dentro de la investigación de la ciencia política y de las relaciones internacionales se desarrollan corrientes feministas en las universidades anglosajonas explorando, desde distintos en-

2 Un ejemplo de ello es que para “enero de 2014 había 46 países que tenían más del $30 \%$ de las mujeres parlamentarias en al menos una de las cámaras. Ahora hay más mujeres al frente de las carteras ministeriales consideradas duras, como Defensa y Relaciones Exteriores" (PNUD, 2014, p. 4).

3 El término es acuñado en el estudio de las relaciones internacionales por el politólogo norteamericano Joseph Nye en su obra Bound to Lead: The changing nature of American Power, escrito en 1990. Nye señala cómo, a través de aspectos culturales, diplomáticos e ideológicos, los Estados también pueden ejercer influencia sobre otros, a esto lo denomina soft power. Paulatinamente, el término soft se fue usando para hacer referencia a temas de la agenda internacional que no representaban amenazas para los Estados y que se relacionaban con el desarrollo. 
foques, campos de estudio y de análisis que permitieran incluir las relaciones sociales de género como categoría analítica.

Desde entonces, el feminismo como movimiento social y como enfoque de las ciencias sociales, se ha posesionado a través de la mirada de la teoría crítica. El feminismo ve la necesidad de transformar relaciones sociales y de empoderar a la mujer para que alcance su plena liberación. A lo largo de su evolución teórica, el feminismo ha ido ampliando su espacio de discusión y de análisis, cuestionando incluso a otros enfoques de las ciencias políticas que no incorporan dentro de su estudio la categoría de género.

En un primer término, la tarea de las investigadoras sociales en los países occidentales se centró en deconstruir lo femenino.

El primer propósito de los estudios de género o de la teoría feminista es desmontar el prejuicio de que la biología determina lo "femenino", mientras lo cultural o humano es una creación masculina. Los estudios de género surgen a partir de la década de los setenta en Estados Unidos a consecuencia del resurgir del movimiento feminista [...]. El estudio del género se ha incorporado en las dos últimas décadas a todas las ciencias sociales. Si el género es una construcción cultural, por fuerza ha de ser objeto de estudio de las ciencias sociales. La introducción del análisis feminista en las ciencias sociales ha traído consigo no solo la redefinición de muchos de sus conceptos, sino también la crisis de sus paradigmas (Cobo, 1995, pp. 55-56).

En cuanto al estudio sobre feminismo dentro de las relaciones internacionales, se incorpora el análisis sobre género para estructurar la política internacional alrededor de los temas relacionados con seguridad internacional y economía global para, de esta forma, modificar las inequitativas relaciones estructurales de poder existentes (Tickner, 2008, p. 263).

Los aportes académicos se desarrollan a la par con la existencia de movimientos sociales en favor de la igualdad política de las mujeres, estos movimientos empiezan a ejercer presión sobre las instituciones internacionales con el fin de vincular al debate internacional esta preocupación. Al interior de estas se empieza a discutir la necesidad de implementar políticas más eficaces alrededor de los temas blandos, en este caso sobre igualdad de género, los cuales se incorporan paulatinamente dentro de la agenda internacional.

La década de los setenta no solo se caracterizó por el surgimiento de movimientos sociales a favor de la mujer, sino también por el auge que las Organizaciones No Gubernamentales (ONG) tuvieron a nivel mundial; su voz de protesta ante la creciente preocupación de la sociedad civil por el avance de problemáticas sociales las llevó a participar de distintos encuentros internacionales con un alto contenido social, ejerciendo de este modo mayor presión ante los gobiernos para la puesta en marcha de políticas en pro del desarrollo de la mujer, la protección del medioambiente, el respeto de los derechos humanos, entre otros temas que parecían rezagados en la agenda de los Estados.

En el año de 1975, reunidos en Ciudad de México, representantes de la sociedad civil y de los Estados miembros de la ONU, celebraron la Conferencia Mundial del Año Internacional de la Mujer, en la cual se esta- 
blecieron los lineamientos para el progreso de las mujeres durante los siguientes diez años en materia de empleo, educación y salud, y cuyo seguimiento se hizo durante las conferencias de Copenhague (1980), Nairobi (1985) y Beijing (1995), en esta última se aprueba una plataforma de acción ${ }^{4}$.

En 1981, entra en vigor la Convención sobre la Eliminación de todas las formas de discriminación contra la mujer (Cedaw), la cual señala que "la máxima participación de la mujer, en igualdad de condiciones con el hombre, en todos los campos, es indispensable para el desarrollo pleno y completo de un país, el bienestar del mundo y la causa de la paz"', reconociendo de este modo la importancia que tiene para el desarrollo el empoderamiento de las mujeres, o también, como posteriormente lo llamaría el economista Amartya Sen, el desarrollo de sus capacidades ${ }^{6}$.
El fin de la Guerra Fría permitió que se priorizaran temas distintos a los de seguridad, como por ejemplo, las cuestiones sobre medioambiente, desarrollo, derechos humanos y género. En cuanto a este último, podrían señalarse dos importantes acciones, que aunque no son las únicas, evidencian el interés de la comunidad internacional por trabajar en pro de la igualdad de género y del empoderamiento de la mujer. En primer lugar, la creación en 2010 de onu Mujeres, con el mandato de apoyar, asistir, dirigir y coordinar las acciones del sistema de Naciones Unidas y, por ende, de los Estados, en la eliminación de la discriminación en contra de las mujeres y las niñas; en empoderar a la mujer y alcanzar la igualdad entre mujeres y hombres ${ }^{7}$.

En segundo lugar, la construcción de una agenda global para el desarrollo que se estableció en el año 2000 , con la firma de la

4 "La Plataforma de Acción hace hincapié en que las mujeres comparten problemas comunes que solo pueden resolverse trabajando de consuno y en asociación con los hombres para alcanzar el objetivo común de la igualdad de género en todo el mundo. La Plataforma respeta y valora la plena diversidad de las situaciones y condiciones en que se encuentra la mujer y reconoce que algunas mujeres enfrentan barreras especiales que obstaculizan su participación plena y en pie de igualdad en la sociedad". Para mayor información sobre la resolución aprobada de la Declaración y Plataforma de Acción de Beijing, celebrada en septiembre de 1995, véase http://www.un.org/womenwatch/daw/ beijing/pdf/BDPfA\%20S.pdf

5 Para mayor información, se puede acceder en línea a la Convención sobre la eliminación de todas las formas de discriminación de la mujer, en: http://www.un.org/womenwatch/daw/cedaw/text/sconvention.htm

6 "En el análisis del desarrollo, las libertades de los individuos constituyen la piedra angular. Por tanto, prestamos especial atención a la expansión de capacidades de las personas para llevar el tipo de vida que valoran y que tienen razones para valorar" (Sen, 2000, p. 34).

7 Con el fin de no duplicar esfuerzos y de trabajar hacia el mismo objetivo, onu mujeres continúa con el trabajo que venían realizando otras entidades en las Naciones Unidas como: La División para el Adelanto de la Mujer (DAW), el Instituto Internacional de Investigaciones y Capacitación para la Promoción de la Mujer (INSTRAw), la Oficina del Asesor Especial en Cuestiones de Género (OSAGI) y el Fondo de Desarrollo de la Mujer (UnifEm). Toda la información respecto al funcionamiento y los trabajos que actualmente realiza onu mujeres alrededor del mundo puede consultarse en: www.unwome.org 
Declaración del Milenio, a través de la cual se establecieron los Objetivos de Desarrollo del Milenio (ODM), cuya principal meta era disminuir la pobreza extrema y el hambre en quince ańos; para ello, los países signatarios se comprometían a implementar a nivel local los ocho objetivos ${ }^{8}$.

Dentro de los ocho objetivos establecidos, dos de estos encaminan sus acciones al cumplimiento del mandato general de onU Mujeres. Por un lado, el objetivo 3, estableció como prioritaria la promoción de la igualdad entre los sexos y el empoderamiento de la mujer; la meta establecida para su cumplimiento fue la de "eliminar las desigualdades entre los géneros en la enseñanza primaria y secundaria, preferiblemente para el año 2005, y en todos los niveles de la enseñanza antes de finales de 2015 "9. Por otro lado, el objetivo 5, establecía el mejoramiento de la salud materna, entre cuyas metas estaban la de "reducir un 75\% la tasa de mortalidad materna entre 1990 y 2015" y "lograr, para 2015, el acceso universal y la salud reproductiva".

La inclusión de los temas de género como meta del milenio y como política en lo local, han surgido gracias a la influencia de movi- mientos sociales que nacen por la convicción de la existencia de un sistema patriarcal ${ }^{10} \mathrm{y}$ de una construcción social e histórica que ha girado alrededor del rol del hombre dentro de las estructuras sociales, económicas y políticas.

En el caso de los países desarrollados, se han venido implementando políticas de género que han permitido la paridad al interior de sus Estados. Su experiencia sirve de insumo para que países en desarrollo, por ejemplo, aprendan e implementen políticas más favorables dentro de sus sistemas locales. En cuanto a la protección de los derechos de la mujer, Martha Nussbaum señala en su libro Las mujeres y el desarrollo humano, cómo el sistema jurídico y político de Estados Unidos inspiró la creación de políticas en la India en pro del desarrollo y la protección de la mujer, afirmando que la Constitución de la India es un documento que al final le resulta muy favorable a esta (2002, p. 56).

Lo anterior es un ejemplo de cómo una experiencia local exitosa o eficaz termina siendo transferida a otro sistema; en el caso estudiado por Nussbaum hay una transferencia de ideas e instituciones para el desarrollo de políticas del sistema norteamericano al sistema

8 Para profundizar sobre este tema véase la página de la onu, en http://www.un.org/es/millenniumgoals/

9 Sin embargo, el informe elaborado por el PNUD en 2014 sobre el seguimiento de los oDM señala que "la disparidad entre los géneros predomina en los niveles más altos de la enseńanza y las variaciones más notables se observan en las regiones en desarrollo" (PNUD, 2014, p. 21).

10 Con respecto al patriarcado, Alicia Puleo (1991, pp. 21-54), en Amoros (1995), realiza un completo análisis sobre este término que etimológicamente significa gobierno de los padres. "Serán las feministas radicales quienes se valgan del término patriarcado como pieza clave de sus análisis de la realidad. Con él denuncian una situación sistemática de dominación masculina en la que los hombres particulares aparecen como agentes activos de la opresión sufrida por las mujeres" (p. 23). 
de la India. En cuanto al concepto de policy transfer o transferencia de politicas ${ }^{11}$, este es un concepto desarrollado en la década de los noventa que se define como "el proceso por el cual el conocimiento acerca de las políticas, arreglos administrativos, instituciones e ideas en un sistema político (pasado o presente) es usado en el desarrollo de políticas, arreglos administrativos, instituciones e ideas en otro sistema” (Dolowitz y Marsh, 2000, p. 5).

Sin embargo, el análisis de Nussbaum también puede servir de ejemplo para señalar que el arraigo de las costumbres o los aspectos culturales puede ejercer una fuerte incidencia, en el éxito o no, de la transferencia de políticas. En el estudio de caso abordado por Nussbaum, se evidencia que en la práctica no existe la misma favorabilidad que se consagra en la constitución para la protección de los derechos de la mujer. "La India nunca entendió la igualdad de la manera meramente formal que ha prevalecido algunas veces en la ley de los Estados Unidos, aquí hubo un entendimiento compartido de que la igualdad posee requisitos materiales e institucionales" (2002, p. 56), lo que demuestra este caso es que el tema de género, mujer e igualdad debe superar una prueba más profunda, la cultural.

Por tanto, el aprendizaje de dichas experiencias tanto internacionales como locales dentro del proceso de identificación de un problema público, podría llegar a mejorar la implementación de políticas de un sistema a otro. Por lo cual, la importancia de incorporar este análisis dentro de un marco de gobernanza; la transferencia debe darse, no solo en la incorporación o adecuación de modelos, sino también del aprendizaje y de la participación de distintos agentes, los cuales puedan obtener experiencias agregadas.

Un ejemplo en concreto sobre la transferencia de política globales de género está relacionado con la construcción de marcos jurídicos que permitan a los gobiernos proteger la vida e integridad de la mujer. El informe Femicidio en América Latina y el Caribe (Garita, 2011), desarrollado en el marco de la campaña del Secretario General de la ONU, Ban Ki-moon: "UnÉTE para poner fin a la violencia contra las mujeres en América Latina y el Caribe", estudia el caso de seis países de la región (Chile, Costa Rica, El Salvador, Guatemala, México, Nicaragua y Perú) que a 2012 habían implementado leyes contra el femicidio y otras formas de violencia contra las mujeres.

Dentro de este proceso de policy transfer, pueden participar diversos actores; Dolowitz y Marsh, por ejemplo, identifican nueve categorías (cuadro 3). La multiplicidad de actores lleva necesariamente a enmarcar el análisis dentro del concepto de gobernanza, por el cual se entenderá:

Proceso de coordinación de actores, grupos sociales, instituciones, con el ánimo de alcanzar objetivos definidos y discutidos colectivamente [...] Conjunto de instituciones, de redes, directivas, reglamentos, normas,

11 En este contexto, por policy se hace referencia a la política pública como programa de acción. Ver Pierre Muller (2002). 
usos políticos y sociales, así como actores públicos y privados que contribuyen a la estabilidad de una sociedad y de un régimen político, orientación, capacidad de dirigir y de proporcionar servicios y asegurar la legitimidad [...]. Gobernanza se refiere a la formación de reglas que reducen los costos de transacción y mejoran la eficiencia de las políticas públicas mediante la organización de la competencia entre agencias (Ravinet, 2009).

Para entender la definición anterior podrían resaltarse los casos de la Organización para la Cooperación y el Desarrollo EconómiCo (OCDE), al igual que el de la Unión Europea, dos claros ejemplos de cómo las organizaciones internacionales sirven de plataforma para facilitar la transferencia de políticas, sobre todo en materia de cooperación internacional para el desarrollo (Conde, 2005, p. 3) ${ }^{12}$, especialmente, como lo señalan Dolowitz y Marsh, en el desarrollo de ideas, programas e instituciones alrededor del mundo. Organizaciones como las Naciones Unidas, a través de sus distintas agencias, ejercen influencia en la formulación de políticas en el plano local de los Estados y apoyan su implementación y posterior evaluación. Por su parte, las ONG también incrementan su influencia para el diseño de políticas globales (2000, p. 11) e incluso para la apuesta en agenda por parte de entidades locales.

"El desarrollo de las intervenciones del Estado en la sociedad civil es, sin duda alguna, uno de los mayores fenómenos del siglo xx. No solamente los campos de acción de la administración se han ampliado y multiplicado, también las técnicas utilizadas por los decisores públicos se han diversificado y sofisticado" (Muller, 2002, p. 29). La existencia de campos cada vez más complejos de interacción (entre sociedad civil y Estado), necesariamente lleva a la búsqueda de escenarios que permitan resolver los problemas colectivos dentro de marcos de gobernanza global.

\section{EL DESARROLLO TEÓRICO DEL FEMINISMO}

Durante las décadas de los sesenta y setenta, tanto en Estados Unidos como en Inglaterra, el feminismo empezó a incorporarse dentro de la agenda de lo político cuando se construyó la idea de que lo personal traspasaba al plano de lo político ${ }^{13}$. Por su parte,

12 Un ejemplo señalado por el autor es la cooperación que existe entre la Unión Europea y los países de Oriente Medio y del Norte de África (MENA, por sus siglas en inglés), en la cual ha sido clave la transferencia de políticas a través de completos programas de cooperación. "La Unión Europea es por tanto un ejemplo de cómo sistemas complejos de gobernanza, en los cuales participan un gran número de actores, se interconectan en procesos de mutua interacción a partir de procesos de cooperación en tres frentes: cooperación política y de seguridad, cooperación económica y financiera y cooperación social y cultural" (Conde, 2005).

13 Jenny Chapman inicia su análisis sobre Perspectiva Feminista haciendo un estudio sobre el feminismo radical que se desarrolló en la década de los sesenta, esta etapa transformó la noción del feminismo más allá de un movimiento social, y lo legitimó dentro de un análisis teórico con influencia en temas políticos, de ahí la afirmación que la autora hace sobre la idea de que lo "personal es político", temas privados entran en la agenda de lo público (1995, p. 103). 
Jacqui True (2009) agrega que si es político es también internacional, pues las relaciones de género forman una parte integral de las relaciones internacionales.

El feminismo, por ejemplo, ha servido como punto de referencia para la construcción metodológica de la investigación en ciencias sociales. Marsh y Stoker, en su libro Teoría y métodos de la ciencia política, identifican seis enfoques los cuales "dan lugar a un amplio abanico de teorías [que de alguna manera permiten] explicar, comprender e interpretar la realidad. De hecho es posible ir más allá y afirmar que sin alguna clase de teoría es imposible entender la realidad" (1995, p. 27). La importancia de señalar esto radica en que un enfoque teórico facilita el intercambio de ideas, fomenta nuevas investigaciones y permite que ese conocimiento sea de dominio público y lleve a la sociedad a un proceso de entendimiento sobre sí misma, de análisis o de cambio ${ }^{14}$. El feminismo, por tanto, hace parte de estos enfoques. El cuadro 1 es elaborado por los autores para explicar el alcance de esté dentro de los procesos investigativos:

\section{Feminismo y relaciones internacionales}

Para el desarrollo de este punto, se incluirán los trabajos de investigación sobre feminismo y género que desde las relaciones

\section{CUADRO 1. FEMINISMO COMO ENFOQUE DE CIENCIA POLÍTICA DE MARSH Y STOKER (1995, p. 23)}

\begin{tabular}{|c|l|}
\hline Objeto de estudio & $\begin{array}{l}\text { Analiza el impacto del patriarcado } \\
\text { con el propósito de cuestionarlo. }\end{array}$ \\
\hline $\begin{array}{c}\text { Orientación } \\
\text { metodológica }\end{array}$ & $\begin{array}{l}\text { Relativista, a favor de los métodos } \\
\text { cualitativos. Ataca los métodos } \\
\text { cuantitativos apelando a una me- } \\
\text { todología feminista alternativa. }\end{array}$ \\
\hline $\begin{array}{c}\text { Naturaleza de la } \\
\text { teoría }\end{array}$ & $\begin{array}{l}\text { Normativo, prescriptivo, evaluati- } \\
\text { vo, empírico. }\end{array}$ \\
\hline $\begin{array}{c}\text { Concepción del } \\
\text { Estado y de la } \\
\text { política }\end{array}$ & $\begin{array}{l}\text { Persigue la feminización del deba- } \\
\text { te y la ampliación de la definición } \\
\text { de lo político. }\end{array}$ \\
\hline $\begin{array}{c}\text { Posición dentro } \\
\text { de la disciplina }\end{array}$ & $\begin{array}{l}\text { Enfoque reconocido de impacto } \\
\text { limitado. Su potencial es grande } \\
\text { si prescinde de los excesos del } \\
\text { posmodernismo. }\end{array}$ \\
\hline
\end{tabular}

internacionales han elaborado: J. Ann Tickner (2006 y 2008), Terrel Carver, Marysia Zalewski, Helen Kinsella y Charli Carpenter (2003), Jacqui True (2009), Gillian Youngs (2004), Mónica Salomón (2002).

El feminismo entró al estudio de las relaciones internacionales a partir de la década de los ochenta, y con mayor fuerza luego del fin de la Guerra Fría, cuando se introduce el análisis de género dentro de la disciplina, en especial cuando se empieza a prestar mayor atención a los conflictos étnicos del orden nacional y el número de víctimas de estos,

14 Dentro de estos enfoques se encuentran: la teoría normativa, el institucionalismo, el análisis conductista, la teoría de la elección racional, el análisis del discurso y el feminismo. "Cada uno de ellos tiene una orientación metodológica determinada" (Marsh y Stoker, 1995, p. 25) y "responden a preguntas sobre el objeto principal del que ha de tratarse; el método para obtener datos y la naturaleza del proceso de teorización que debe llevarse a cabo, además de mostrar diferentes presupuestos subyacentes en el carácter y funcionamiento de la política” (p. 19). 
que en su mayoría eran mujeres. Tickner (2008) señala la importancia que los movimientos sociales y las ONG tuvieron dentro de las organizaciones internacionales para la formulación de políticas sobre los temas blandos de la agenda.

Las autoras mencionadas anteriormente coinciden en señalar que el análisis de género, en especial en países occidentales, busca responder preguntas acerca de la estructura, es decir, de la jerarquía predominante dentro del sistema internacional; su propuesta, por tanto, se enmarca en la teoría crítica ${ }^{15}$ y se consolida como parte de los "enfoques disidentes" cuyo objetivo es "acabar con las situaciones de desigualdad, explotación y opresión de la mujer" (Salomón, 2002, p. 32).

A partir de entonces, se multiplican las investigaciones en temas de género que utilizan este enfoque como herramienta analítica para entender las relaciones de poder global imperantes, así como mirar el impacto que este tiene sobre el comportamiento de los Estados, el conflicto internacional y sobre fenómenos de paz y de guerra usando el género como variable de análisis cuantitativa (True, 2009, p. 237).

En esencia, las feministas no están satisfechas con la composición de la política internacional en términos de las políticas interestatales, tal como lo señala Tickner
(2008). Cuando las mujeres participan en la política internacional lo hacen más a través de movimientos sociales o no gubernamentales. Las mujeres son la mayoría de la población pobre y las políticas económicas se construyen de manera distante, en los centros de poder, determinando cómo afectará a las políticas locales y de recursos (p. 264).

Las teorías feministas dentro del estudio de las relaciones internacionales incorporan el concepto de "género" como instrumento de análisis para comprender las relaciones de poder, así como las jerarquías del sistema, y, para definirlo, parten del análisis sobre poder, autonomía, racionalidad y lo público, conceptos asociados con masculinidad. Conceptos opuestos a estos son dependencia, debilidad, conexión, emotividad y privacidad, que son asociados con lo femenino. (True, 2009, p. 237)

Desde esta perspectiva, el género es crucial para analizar la política y la economía global, particularmente respecto a los temas de inequidad, inseguridad y justicia social, en un sistema jerárquico en donde las características de la masculinidad son más valoradas que las de la feminidad (Tickner, 2008, p. 265).

Jacqui True señala que el feminismo ha hecho importantes aportes a las relaciones internacionales, dichos aportes los analiza a través de tres perspectivas (2009, p. 238) que

15 Las aproximaciones teóricas feministas entran al campo de las Relaciones Internacionales al mismo tiempo que se da lo que se conoce como el "tercer debate" o "debate interparadigmático" que se dio entre los paradigmas realistas, transnacionalistas y estructuralistas "a partir de la introducción en relaciones internacionales de la noción kuhniana de paradigma cientifico" (Salomón, 2002, p. 9). 
favorecen la comprensión y el estudio del feminismo, y sirven además de insumo teórico para el diseño y puesta en marcha de políticas en un marco de gobernanza:

1. Feminismo empírico: se enfoca en la mujer, explora el género como una dimensión empírica de las relaciones internacionales. Desde acá se logran documentar los efectos de procesos que, como la globalización, han incrementado la desigualdad entre hombres y mujeres; sus estudios han revelado la "feminización de la pobreza", la cual es desproporcionada entre hombres y mujeres. Los estudios de Tickner apuntan a señalar que aún hoy, en pleno siglo XXI, siguen existiendo inequitativas estructuras económicas que establecen desiguales relaciones de poder entre hombres y mujeres.

2. Feminismo analítico: usa al género como una categoría teórica.

3. Feminismo normativo: refleja el proceso de teorización como parte de la agenda normativa para lograr cambios globales tanto en lo social como en lo político.

La investigación feminista ha visibilizado el rol de las mujeres como principales proveedoras y productoras de necesidades básicas, en especial de las mujeres en países en desarrollo, mostrando la importancia de empoderarlas en el plano local. Jacqui True, señala en su análisis que a través de estudios empíricos se ha demostrado que empoderando a la mujer estas pueden ser generadoras de crecimiento social. Señala, a manera de ejemplo, cómo en el sector agrícola la asignación de recursos de cooperación o la financiación de créditos destinados a las mujeres las empodera y las vuelve parte activa del proceso de mejorar su calidad y nivel de vida.

Bangladesh, por ejemplo, adelantó este proceso con la creación del Banco Grameen (Banco Rural), que nace gracias a la idea del economista Muhammad Yunus de otorgar pequeños préstamos a mujeres de escasos recursos con el objetivo de apoyar proyectos emprendedores, un claro ejemplo de transferencia de políticas que se implementa alrededor del mundo.

Lo interesante de estos enfoques desarrollados por True es que a lo largo de la historia, no solo se ha victimizado a la mujer, también se han logrado avances gracias al desarrollo tecnológico que no solo evidencia sus problemáticas sino que les brinda espacios de actuación y de comunicación. Por ejemplo, las feministas liberales exploran cómo el comercio y la liberalización de la mujer pueden transformar acciones locales.

Lo anterior hace mención a la división que al interior de la corriente feminista se da en la década de los noventa por las diferencias que se presentan entre estas: feministas lesbianas, feministas de países en desarrollo, feministas negras, feministas blancas, feministas pobres, etc. A medida que la agenda sobre los temas blandos se expande, las diferencias entre estas también. Aunque la investigación sobre feminismo y relaciones internacionales ha avanzado, sus estudios siguen centrándose en la mujer, lo que refuerza o limita el alcance de sus investigaciones, limitándolas 
a una noción política que solo busca dar fin a la opresión ${ }^{16}$.

Por otra parte, para su estudio, el feminismo ha tomado elementos teóricos desarrollados en el campo de las relaciones internacionales para comprender y facilitar su estudio, desde una mirada liberal, marxista y radical, como las principales, pero que para otros autores incorpora un análisis posliberal, constructivista, posmoderno y poscolonial. Desde diversas miradas teóricas, las feminis- tas buscan producir conocimiento para que a distintos niveles (público, privado, global o local) se construyan prácticas que mejoren la calidad de vida de las mujeres. Por tanto, su objetivo (Tickner, 2008; True, 2009) va encaminado a explicar la subordinación de las mujeres, la cual existe en distintos grados en todas las sociedades y de este modo explorar maneras de terminarla.

El cuadro 2 sintetiza los aportes que desde distintas teorías se hacen al feminismo.

\section{CUADRO 2. FEMINISMO Y RELACIONES INTERNACIONALES}

\begin{tabular}{|c|c|c|}
\hline $\begin{array}{c}\text { ¿Qué busca el } \\
\text { feminismo dentro } \\
\text { de las relaciones } \\
\text { internacionales? }\end{array}$ & $\begin{array}{l}\text { - Explicar o dar respuesta a la subordinación de las mujeres en la política internacional (Tickner, } \\
\text { 2008) introduciendo el género como categoría de análisis. }\end{array}$ \\
\hline Permite reconocer dinámicas de poder de los Estados-nación que tienen incidencia en el \\
orden mundial, en su estructura (True, 2009, p. 259).
\end{tabular}

16 A este respecto, es interesante revisar el análisis que a través de varios artículos Terrel Carver, Marysia Zalewski, Helen Kinsella y Charli Carpenter (2003) hacen en su artículo “Gender and International Relations”, en el cual realizan un análisis más crítico sobre las limitaciones que los estudios sobre género, incorporados a la luz de las Relaciones Internacionales, han desarrollado. 


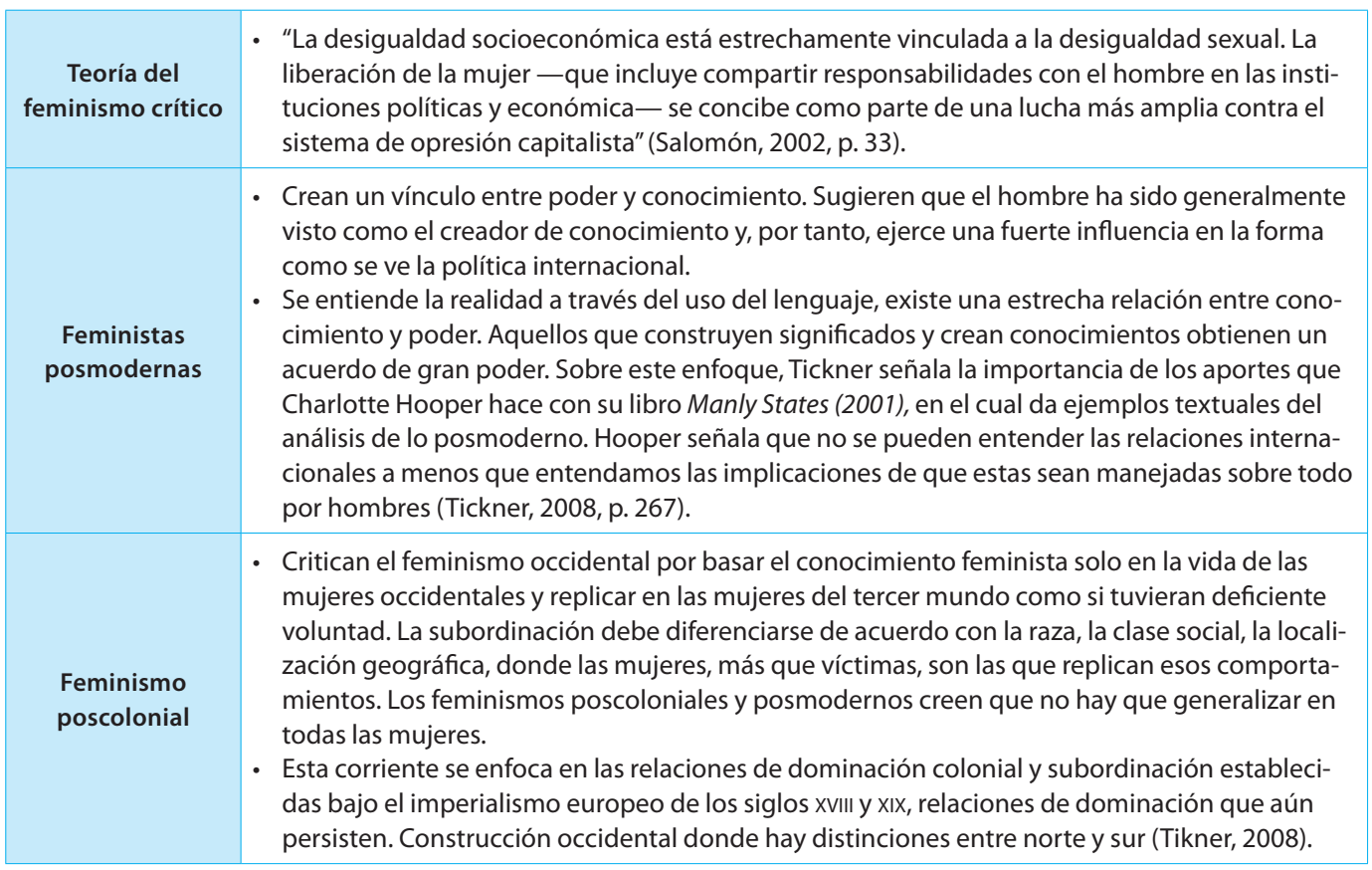

Fuente: elaboración propia a partir de Tickner (2008), Salomón (2002) y True (2009).

\section{LA TRANSFERENCIA DE POLÍTICAS Y SU PUESTA EN AGENDA}

"Política no es solo una decisión. Es ante todo acción, un conjunto de acciones" (Aguilar, 1992).

La trasferencia de politicas no es algo exclusivo del campo de estudio del análisis de las políticas públicas (APP), por su carácter interdisciplinar involucra el análisis desde distintas disciplinas, por ejemplo, las relaciones internacionales. Dentro del proceso de transferencia se involucran distintos actores. A nivel global, organizaciones internacionales como la ONU, la OCDE, el Fondo Monetario Internacional, el Banco Mundial, y, como se mencionó, la Unión Europea (Evans, 2009, p. 243$)^{17}$ desempeñan un papel fundamental en el diseño e intercambio de ideas, políticas, instituciones, etc.

En el marco de la ONU, en 2010 la Asamblea General aprobó la creación de ONU Mu-

17 Algunos de los aportes que se hacen desde las relaciones internacionales para la transferencia de políticas es el uso de términos que permiten identificar distintas formas dentro del proceso de transferencia, como lo es el bandwagoning. Entre otras formas están: diffusion, emulation, policy learning, social learning y lesson-drawing (Evans, 2009, p. 244). 
jeres, un órgano encargado de acelerar el progreso en el logro de la igualdad de género y el empoderamiento de las mujeres ${ }^{18}$, así como de apoyar la labor de los países a nivel local respecto al cumplimiento del oDM 3 , el cual hace referencia a la promoción de la igualdad entre los sexos y el empoderamiento de la mujer, un ejemplo sobre la transferencia global de políticas de género.

La mundialización de los procesos de desarrollo se vislumbran en los acuerdos a los cuales llegan los Estados a través de sus compromisos internacionales. "En esta época de mundialización e interdependencia hay un aumento de sistemas políticos, económicos y sociales tanto nacionales como supranacionales, el asunto de la importación y exportación de modelos se instaura de manera más intensa" (Dumoulin y Saurugger, 2010, p. 9) ${ }^{19}$.

Tal como seńalan distintos autores ${ }^{20}$, el policy transfer es una práctica que se ha favorecido gracias al acelerado crecimiento de tecnologías de la información que han facilitado la comunicación, así como el intercambio de ideas y de conocimiento entre los policy-makers de distintas partes del mundo, pues ningún país hoy en día se encuentra totalmente ajeno de saber qué pasa en otros lugares. El desarro- llo de la política pública ahora se posiciona en un ámbito global (Dolowitz y Marsh, 2000, p. 6).

Dolowitz y Marsh (2000) desarrollaron un modelo que permite entender cómo se da la transferencia de políticas públicas. Dicho modelo se estructura alrededor de seis preguntas básicas que buscan facilitar tanto el estudio como el análisis de las experiencias, las cuales se resumen en el cuadro 3 .

\section{La puesta en agenda: las políticas de género}

Hasta el momento se ha señalado en qué medida la transferencia de políticas se ha venido gestando, por ejemplo, en las políticas de género. Retomando la definición dada inicialmente de Dolowitz y Marsh, ese conocimiento acerca de las políticas, los arreglos administrativos, las instituciones y las ideas desarrollado, en el marco de la ONU, son un claro ejemplo de cómo se han construido políticas que han permitido algunos avances.

Pero para que dichos procesos se lleven a cabo, es importante pensar en cómo se dará esa puesta en agenda. El polítologo francés Pierre Muller y uno de los pioneros alrede-

18 Actualmente, onU-Mujeres tiene presencia en cinco continentes y su objetivo es el de "promover el empoderamiento y los derechos de las mujeres y la igualdad de género a nivel global y al interior de los países por medio de una red de oficinas subregionales, de país y de enlace. [...] Según la resolución 64/289 de la Asamblea General de la ONU, la organización está regida por una estructura de gobernanza intergubernamental”. Para mayor información sobre los trabajos que ONU-mujeres adelanta a nivel local, véase www.unwomen.org/es.

19 Traducción propia a partir del original en francés.

20 Para más información sobre las causas que llevan a un incremento de la práctica de transferencia de politicas, véanse los textos de Dolowitz y Marsh (2000), Dumoulin y Saurugger (2010) y Rose (1991). 


\section{CUADRO 3. ESTRUCTURA DE TRANSFERENCIA DE POLÍTICAS DE DOLOWITZ Y MARSH}

\begin{tabular}{|c|c|}
\hline Pregunta & Análisis de los autores \\
\hline $\begin{array}{l}\text { 1. ¿Quiénes participan del } \\
\text { proceso de transferencia de } \\
\text { políticas? }\end{array}$ & $\begin{array}{l}\text { Los autores identifican nueve categorías de funcionarios electos: partidos políticos, } \\
\text { burócratas/funcionarios, grupos de presión, empresarios políticos y expertos, corpora- } \\
\text { ciones transnacionales, centros de pensamiento, instituciones supranacionales e insti- } \\
\text { tuciones no gubernamentales y finalmente consultores (Dolowitz y Marsh, 2000, p. 10). } \\
\text { En cuando a las políticas de género, la construcción de un ideario donde se señala que } \\
\text { es importante que la mujer alcance una paridad con los hombres para mejorar su ca- } \\
\text { lidad de vida, así como el desarrollo social, ha involucrado necesariamente a todos los } \\
\text { actores señalados por Dolowitz y Marsh. }\end{array}$ \\
\hline 2. ¿Qué se tra & $\begin{array}{l}\text { "Casi cualquier cosa puede ser transferida de un sistema político a otro, dependiendo } \\
\text { del tema o de la situación [...] se identifican ocho categorías distintas: metas políticas, } \\
\text { contenidos políticos, instrumentos, programas, instituciones, ideologías, ideas y actitu- } \\
\text { des e incluso lecciones negativas" (Dolowitz y Marsh, 2000, p. 12) }{ }^{21} \text {. } \\
\text { En el caso de la transferencia de políticas globales de género, en la introducción se } \\
\text { señaló el rol que las organizaciones internacionales han desempeñado en el diseño } \\
\text { y construcción de ideas y actitudes que se adecuaran a los programas y contenidos } \\
\text { políticos en el orden local. Pero a su vez, esta transferencia se da, gracias a la presión } \\
\text { proveniente de movimientos sociales, academia, ong e individuos a partir de la década } \\
\text { de los setenta en algunos países occidentales. }\end{array}$ \\
\hline $\begin{array}{l}\text { 3. ¿De dónde se transfieren } \\
\text { las políticas? }\end{array}$ & $\begin{array}{l}\text { Se ha venido construyendo la idea de que las policy transfer deben construirse dentro } \\
\text { de marcos de gobernanza, pues pueden provenir del plano internacional, nacional o } \\
\text { local. }\end{array}$ \\
\hline $\begin{array}{l}\text { 4. ¿Cuáles son los distintos } \\
\text { tipos de transferencia? }\end{array}$ & $\begin{array}{l}\text { Lo interesante que plantea la transferencia de políticas es que no es un juego de suma } \\
\text { cero en donde debe transferirse todo o nada. Cada caso es particular y puede envolver } \\
\text { distintas combinaciones, tanto de procesos como de agentes. Los autores señalan cua- } \\
\text { tro tipos de transferencias, a saber: copying (completa y directa transferencia); emula- } \\
\text { tion (transferencia de ideas detrás de un programa o política); combinations (envuelve } \\
\text { procesos mixtos de distintas políticas); inspiration (como una política en otra jurisdic- } \\
\text { ción puede inspirar cambio, el resultado final no necesariamente responde al original). }\end{array}$ \\
\hline $\begin{array}{l}\text { 5. ¿Qué facilita o limita el } \\
\text { proceso? }\end{array}$ & $\begin{array}{l}\text { Respecto a lo que facilita el proceso de policy transfer, Diana Stone (2001) señala como } \\
\text { aspecto fundamental la construcción de redes, las cuales pueden facilitar el proceso de } \\
\text { intercambiar ideas, normas e información. Sin embargo, también se identifican variables } \\
\text { que pueden llegar a limitar la transferencia, uno de los casos que se ha venido mencio- } \\
\text { nando es el tema cultural. (Evans, } 2009 \text { señala otras tres limitaciones: 1) lo que sucede } \\
\text { durante la etapa de predecisión; 2) el ambiente, que puede estar ligado al tema cultural; } \\
\text { 3) la opinión pública, la cual puede ejercer fuerte influencia para su implementación o no. }\end{array}$ \\
\hline $\begin{array}{l}\text { 6. ¿Cómo se relaciona con el } \\
\text { éxito o fracaso de la política? }\end{array}$ & $\begin{array}{l}\text { Se debe partir del hecho de que no todas las políticas terminan siendo exitosas. Por lo } \\
\text { cual, es importante analizar qué factores podrían incidir en el éxito o el fracaso de una } \\
\text { política. }\end{array}$ \\
\hline
\end{tabular}

Fuente: elaboración propia a partir del modelo propuesto por Dolowitz y Marsch (2000).

21 "We identify eight different categories: policy goals, policy content, policy instruments, policy programs, institutions, ideologies, ideas and attitudes and negative lessons” (Dolowitz y Marsh, 2000, p. 12). 
dor del estudio y construcción teórica de las políticas públicas, parte de preguntarse "¿cómo se toman las decisiones que constituyen un programa de acción gubernamental?, que es el problema del decision making [...] y, ¿cómo se aplican las decisiones por parte de los organismos encargados de ejecutarlas?, que vendría siendo el problema de la puesta en marcha o implementation" (2002, p. 55), preguntas que se plantea ante la presencia, y cada vez mayor influencia que ejercen actores distintos al Estado.

Por tanto, la definición por la cual se entenderá política pública será la propuesta por Pierre Muller, quien señala que un asunto o cuestión se vuelve objeto de una política pública porque hay un problema para resolver (2002, p. 63), es decir, que "en una sociedad dada, cualquier problema social es susceptible de volverse política pública” (p. 64).

La definición anterior sugiere que la formulación de una política pública puede ser de doble vía, lo cual se refleja en el proceso de transferencia de políticas, pues estas se pueden construir a través de la intervención del Estado o "modelo de oferta administrativa", en el cual son las instituciones de este las encargadas de inscribir los temas en la agenda y, por otro lado, está presente aquella que proviene de una fuente externa al Estado como por ejemplo grupos sociales, a este tipo de intervención se le denomina el "modelo de la demanda" (Roth, 2002).

La anterior definición deja claro que distintos grupos participan en el proceso de la formación de políticas, pues la multiplicación e incorporación de nuevos asuntos hace que los temas públicos excedan la capacidad de los tomadores de decisión, que es lo que se ha venido mencionando respecto a las policy transfer. Dentro de este análisis, el artículo de Roger Cobb, Jennifer Ross y Marc H. Ross "Agenda building as a comparative political process" (1976), señala que la construcción de la agenda requiere comprender cómo distintos grupos participan de las discusiones de temas que, aunque son políticos, terminan incidiendo dentro de la puesta en agenda ${ }^{22}$.

A mediados del siglo xx, la academia norteamericana fortalece programas en el campo de las ciencias sociales encaminados a comprender el quehacer del Estado. Las ciencias sociales, por tanto, adquieren mayor importancia no solo para los académicos sino también para los políticos. En la década de los sesenta, el interés por el Estado, su funcionamiento y sus instituciones llega más allá de las universidades; para Roth (2002), durante este

22 "Tratándose tanto de los Estados-naciones como de las entidades territoriales, comprende el conjunto de los problemas percibidos como aquellos que piden un debate público e incluso la intervención de las autoridades legítimas $[\ldots]$ un problema deberá responder a tres caracteres para tener alguna posibilidad de ser inscrito en la agenda política: 1. Unas élites [...] unos ciudadanos que pueden estar más o menos organizados, definen una situación como problemática; [...] 2. Este descubrimiento de un problema se acompańa de procedimientos de etiquetaje que lo califican como perteneciente a la esfera de competencia de las autoridades públicas; 3 . Se espera la intervención de la sociedad política, incluyendo la opción de no hacer nada" (Jean-Gustave Padioleau citado por Muller, 2002, p. 66). 
periodo se despierta un verdadero interés por el estudio de la acción pública.

Una característica importante de estas dos décadas fue la presencia de los Welfare States, también conocidos como Estados de bienestar o Estados providencia, cuyo objetivo era la intervención de estatal en todos los asuntos de la sociedad, una especie de Estado asistencialista.

Meny y Thoenig, en su obra más representativa, Politiques Publiques, señalan el proceso mediante el cual los Estados de bienestar lograron alcanzar su máximo auge durante los periodos que precedieron a la Segunda Guerra Mundial. Los aspectos económico y fiscal eran los que permitían al Estado asegurar la reproducción social de las necesidades del capitalismo (1992, p. 21), así como la formación, la vivienda y la protección social de los trabajadores y de sus familias garantizando al mismo tiempo la ley y el orden (p. 22).

A los Estados de bienestar se les atribuye la aparición del campo de las políticas públicas debido al incremento del intervencionismo estatal en la mayoría de aspectos de la vida social (Roth, 2002, p. 11). Escenario propicio para el desarrollo de los primeros movimientos sociales en favor de la mujer que exigían una mayor intervención del Estado para garantizar y proteger sus derechos como ciudadanas, en especial en lo que respecta a las políticas de asistencia social para disminuir la doble jornada, políticas para mejorar sus condiciones laborales y salariales, y las más recientes, encaminadas a proteger la dignidad y la vida de las mujeres.

Ya entrada la década de los ochenta, los analistas de políticas públicas ganaban un mayor espacio dentro del diseño de las policies, creándose a lo largo de las universidades norteamericanas think tanks o centros de pensamiento, con el objeto de brindar a los tomadores de decisión mayores herramientas e insumos teóricos, o, en últimas, análisis caracterizados por su rigurosidad académica e investigativa que sirvieran de insumo para justificar la intervención del Estado en todos los asuntos sociales, especialmente aquellos considerados como un problema.

Lo anterior demuestra que existen distintas formas de construir un problema. Roth (2002) señala dos importantes procesos; el primero hace referencia a la necesidad de lograr una transformación en la vida cotidiana de los individuos originada por cambios sociales que afectan a grupos sociales. Esto va ligado a la concepción del uso de simbolos positivos y familiares para visibilizar un problema, pues de esta forma se busca generar reacciones favorables en la gente que, de otra forma, prodrían ser hostiles o desfavorables. Esta idea es desarrollada en el artículo de Cobb, Ross y Ross de 1976, que utilizan a manera de ejemplo la expansión de los movimientos feministas de la década de los setenta en Estados Unidos, que enmarcan su causa en términos de igualdad pero ligada a simbolos altamente emocionales con los cuales la sociedad se sintiera identificada.

En segundo lugar, Roth señala una segunda fase que hace referencia al paso de un problema privado a uno social. Una vez reconocido el problema como un problema social y formulado como tal, se busca su institucionalización: es decir, el reconocimiento de la necesidad de una intervención pública (o 
política) que se traduzca en leyes y reglamentos (2002, p. 57-58).

Para finalizar, brevemente se mencionarán los tres modelos señalados por Cobb, Ross y Ross que permiten construir una agenda: 1) Inside Access Model, es decir que la puesta en agenda se da desde instituciones públicas o de grupos con fácil acceso a los tomadores de decisión; 2) Outside Model, grupos fuera del Gobierno la estructuran articulando las quejas sociales; 3) Mobilization Model: políticas y programas que los tomadores de decisión desean mover de una agenda formal (una lista de temas que estos consideran importantes pero no relevantes) a una agenda pública (es decir, aquella que incluye temas de alto interés publico y de mayor visibilidad que requieren de mayor atención).

\section{CONCLUSIÓN}

Cuando el género se volvió una categoría de análisis dentro del estudio de las ciencias sociales y paulatinamente de otras disciplinas, se comprendió que las diferencias y las desigualdades existentes entre hombres y mujeres han sido una construcción social que ha limitado la participación de la mujer en distintos escenarios, coartándola para alcanzar su plena libertad. Esta libertad, según el reconocido economista Amartya Sen, dependerá de variables externas como las instituciones sociales, económicas, así como de los derechos humanos y políticos (2000, p. 19).

Sin embargo, hay que superar barreras culturales que aún no permiten su total desarrollo en la mayoría de países del mundo. Revisar los Informes del PNUD sobre Desarrollo
Humano o sobre los odm es evidenciar que aún falta mucho por recorrer en materia de igualdad de género.

Muchas de las características, e incluso términos, que se estudian dentro de las relaciones internacionales acerca de las dinámicas de poder, funcionamiento del sistema internacional y construcción teórica son asociados con masculinidad y se miden en términos de seguridad nacional, lo cual es cuestionado por las feministas dentro de la disciplina; la seguridad también involucra variables económicas, sociales y físicas de los individuos que tienen que ver con las mujeres (Tickner, 2006).

Gillian Youngs (2004), por ejemplo, señala la existencia de tres fenómenos marcados dentro del funcionamiento de la política internacional: 1) dentro del Estado y el mercado se han masculinizado sus estructuras; 2) la construcción social alrededor del término género, hombres y mujeres, ha oscurecido la construcción social en términos de identidad; 3) hay un dominio prevalente de lo masculino en las agencias políticas y económicas, desconociendo los aportes que las mujeres han hecho tanto en la política como en la economía. Aunque su preocupación final gira alrededor de entender ¿por qué si es un tema tan importante para el estudio de las relaciones internacionales sigue estando tan distante de la vida de las mujeres?, ¿por qué las mujeres no toman conciencia de solo ver su ausencia en la construcción de políticas y toma de decisión en temas de seguridad, militares y diplomáticos?

El género como categoría analítica, abarca su estudio principalmente desde la perspectiva de las mujeres; tanta importancia recobra 
este tema y su rol dentro de distintas esferas o la necesidad de que desarrolle sus capacidades, que la literatura al respecto es amplia y busca explicaciones epistemológicas, ontológicas e históricas a la diferencia entre los sexos. Muestra de ello es Amartya Sen, quien dedica un capítulo de su libro Desarrollo y Libertad a hablar sobre el rol de las mujeres: "La agencia ${ }^{23}$ de las mujeres y el cambio social". "El desarrollo exige la eliminación de las principales fuentes de privación de libertad: la pobreza y la tiranía, la escasez de oportunidades económicas y las privaciones sociales sistemáticas, el abandono en que puedan encontrarse los servicios públicos y la intolerancia o el exceso de intervención de los estados represivos" (Sen, 2000, p. 20).

La transferencia de políticas debe ser innovadora y adaptarse a unas circunstancias y realidades locales que respondan a las necesidades reales de la población a la cual se busca impactar o beneficiar. Instrumentos como la cooperación internacional resultan fundamentales a la hora de transferir políticas. Es tan importante el apoyo financiero que se pueda otorgar, como la asistencia técnica o el intercambio de experiencias y de ideas, las cuales pueden ser cruciales a la hora de hacer efectiva la transferencia $y$, de esta forma, favorecer la adopción e implementación de políticas en entornos donde los aspectos cul- turales son fundamentales para la formulación de políticas, en especial en aquellos países con arraigadas costumbres sociales, religiosas y políticas, en los cuales se puede dificultar la implementación de políticas de género por cuestiones netamente culturales.

Finalmente, se podría concluir que estos nuevos modos de análisis dentro del ser de las ciencias sociales "exigen el uso de la investigación, el análisis y el razonamiento para emprender una reflexión en torno al lugar y el peso de la diferencia (raza, género, sexualidad, clase)" (Wallerstein, 2006, p. 61).

La siguiente cita ayuda a concluir este análisis sobre la cosntrucción de políticas locales de género por la transferencia de buenas prácticas, ideas e instituciones globales:

No hace mucho que la labor de estos movimientos consistía principalmente en trabajar por la mejora del trato que recibían las mujeres, por un trato más justo. Se centraba la atención en el bienestar de las mujeres, algo sin duda muy necesario de corregir. Sin embargo, los objetivos han cambiado y se han ampliado poco a poco; se ha dejado de centrar la atención en el bienestar y se ha incorporado y subrayado el papel activo de la agencia de las mujeres. Las mujeres han dejado de ser receptores pasivos de la ayuda destinada a mejorar su bienestar y son vistas, tanto por los hombres como por ellas mismas, como agentes activos de cambio: como promotores dinámicos de transformaciones sociales que

23 "Agente, persona que actúa y provoca cambios y cuyos logros pueden juzgarse en función de sus propios valores y objetivos, independientemente de que los evaluemos o no también en función de algunos criterios externos. Este estudio se refiere al papel del agente del individuo como miembro político y como participante en actividades económicas, sociales y políticas (que van desde participar en el mercado hasta intervenir directa o indirectamente en actividades individuales o conjuntas en el terreno político y de otros tipos" (Sen, 2000, pp. 35-36). 
pueden alterar tanto la vida de las mujeres comos la de los hombres (Sen, 2000, p. 233).

\section{REFERENCIAS}

Aguilar, L. F. (1992). La Hechura de las politicas. México: Porrúa.

Amoros, C. (ed.). (1995). 10 palabras claves sobre mujer. Pamplona: Editorial Verbo Divino.

Carver, T., Zalewski, M., Kinsella H. y Carpenter C. (2003). Gender and International Relations. International Studies Review, 5 (2).

Chapman, J. (1995). La perspectiva feminista. En Marsh, D. y Stoker, G. (eds.). Teoría y métodos en ciencia politica. Madrid: Alianza Universidad Textos.

Cobb, R., Ross, J. K., Ross, M. H. (1976). Agenda building as a comparative political process. The American Political Science Review, 70 (1).

Cobo, R. (1995). Género. En Amorós, C. (ed.), 10 palabras claves sobre mujer (4 ed., pp. 55-83). Pamplona: Editorial Verbo Divino.

Conde, C. (2005). Policy Transfer in the eu: a model for MENA countries? Experts meeting on "Approaches and methologies for the assessment and transfer of best practices in governance and public administration”. Universidad de Granada. Recuperado de: http://unpan1.un.org/intradoc/ groups/public/documents/un/unpan031601.pdf

Dolowitz, D. P. y Marsh, D. (2000). Learning from Abroad: The role of policy transfer in contemporary policy-making. Governance, 13 (1).

Dumoulin, L. y Saurugger, S. (2010). Les policy transfer studies: Analyse critique et perspectives. Presses de Sciences Po. Critique Internationale, 3 (48).

Evans, M. (2009). Policy transfer in critical perspective. Policy Studies, 30 (3).
Greenberg, M. (2009). The Challenges and Opportunities of Gender Equality in Development. World politics Review.

Marsh, D. y Stoker, G. (eds.) (1995). Teoria y métodos de la ciencia politica. Madrid: Alianza Editorial.

Meny, Y. y Thoening, J.-C. (1992). Las politicas públicas. Barcelona: Ariel.

Muller, P. (2002). Las politicas públicas. Bogotá: Universidad Externado de Colombia.

Nussbaum, M. (2002). Las mujeres y el desarrollo humano. Barcelona: Herder.

Ochman, M. (2006). En busca de una nueva sociedad. Los aportes de la teoría feminista a la reformulación del mundo moderno. Revista desafios (15).

pNud (2014). Objetivos de Desarrollo del Milenio. Informe de 2014. New York: Naciones Unidas (ONU). Recuperado de: http://www.un.org/es/ millenniumgoals/pdf/mdg-report-2014-spanish. pdf.

Ravinet, P. (dir.) (2009). Diccionario de políticas públicas. Bogotá: Universidad Externado de Colombia.

Rose, R. (1991). What is lesson-drawing? Journal of Public Policy, 11 (1).

Roth, A. N. (2002). Políticas públicas: formulación, implementación y evaluación. Bogotá: Ediciones Aurora.

Salomón, M. (2002). La teoría de las relaciones internacionales en los albores del siglo xxi: diálogo, disidencia, aproximaciones. Revista electrónica de estudios internacionales, 4.

Sen, A. (2000). Desarrollo y Libertad. Bogotá: Planeta. Staudt, K. (2008). Women and Gender. En Randall, V. (ed.). Politics in the Developing World. Oxford: Oxford University Press.

Stone, D. (2001). Learning lessons, policy transfer and the international diffusion of policy ideas. CSGR Working Paper (69). 
Tickner, A. (2006). Feminism meets international relations: some methodological issues. En Ackerly, B., Stern, M. y True, J. (eds.). Feminist Methodologies for International Relations. Cambridge: Cambridge University Press.

Tickner, A. (2008). Gender in world politics. En Baylis, J., Smith, S. y Owens, P. (2008). The globalization of world politics: and introduction to international relations. London: Oxford University.

True, J. (2009). Feminism. En Burchill, S. et al. Theories of International Relations. London: Palgrave Macmillan.

Youngs, G. (2004). Feminist international relations: A contradiction in terms? Or: Why women and gender are essential to understanding the world 'we' live in. International Affairs, 80 (1).

Wallerstein, I. (ed.) (2006). Debates en las ciencias sociales, de 1945 hasta el presente. En Abrir las ciencias sociales. México: Siglo xxI editores.

\section{Páginas web}

Banco Mundial. Recuperado de: http://www.bancomundial.org/tema/genero

Garita, A. I. (2011). Informe Femicidio/Feminicidio en América Latina y el Caribe. Organización de Naciones Unidas. Recuperado de: http://www. un.org/es/women/endviolence/pdf/reg_del_femicicidio.pdf

ONU Mujeres. Recuperado de: http://www.unwomen. org/es/about-us/about-un-women

Programa de las Naciones Unidas para el Desarrollo. Recuperado de: http://www.undp.org/content/ undp/en/home/mdgoverview/mdg_goals/mdg1/ 